OPEN ACCESS

Edited by: Arun K. Bhunia,

Purdue University, United States

Reviewed by:

Xiaolong He,

Southern Medical University, China

Maria Guadalupe Vizoso Pinto,

CONICET Higher Institute of Biological

Research (INSIBIO), Argentina

${ }^{*}$ Correspondence:

Yantao Wu

ytwu@yzu.edu.cn

Specialty section:

This article was submitted to

Food Microbiology

a section of the journal

Frontiers in Microbiology

Received: 05 February 2021

Accepted: 23 April 2021

Published: 01 June 2021

Citation:

Guo M, Zhang C, Zhang C.

Zhang $X$ and WU Y (2021)

Lacticaseibacillus rhamnosus

Reduces the Pathogenicity

of Escherichia coli in Chickens.

Front. Microbiol. 12:664604.

doi: 10.3389/fmicb.2021.664604

\section{Lacticaseibacillus rhamnosus Reduces the Pathogenicity of Escherichia coli in Chickens}

\author{
Mengjiao Guo ${ }^{1}$, Congyue Zhang ${ }^{1}$, Chengcheng Zhang ${ }^{1}$, Xiaorong Zhang ${ }^{1}$ and \\ Yantao $W^{1,2 *}$ \\ ' Jiangsu Co-Innovation Center for Prevention of Animal Infectious Diseases and Zoonoses, College of Veterinary Medicine, \\ Yangzhou University, Yangzhou, China, ${ }^{2}$ Joint International Research Laboratory of Agriculture and Agri-Product Safety, \\ Yangzhou University (JIRLAAPS), Yangzhou, China
}

Lacticaseibacillus rhamnosus is a recognized probiotic that is widely used in scientific research and clinical applications. This study found that the Lacticaseibacillus rhamnosus GG (LGG) strain can reduce the adhesion of Escherichia coli (E. coli) to primary chicken intestinal epithelial cells by $75.7 \%$ and inhibit $41.7 \%$ of the E. coli that adhere to intestinal epithelial cells. Additionally, LGG showed strong inhibitory ability on the growth of E. coli, Staphylococcus aureus, Salmonella Paratyphi B, and Salmonella Enteritidis in vitro. Furthermore, the influence of LGG on the growth performance, intestinal flora, immunity, and disease resistance of chickens was explored. Chickens fed with LGG exhibited increased average daily weight gain and concentrations of slgA, IgG, and IgM than did controls. After 21 days of feeding, a diet with LGG increased the diversity of intestinal microbiota and maintained intestinal health. Moreover, LGG promoted immunologic barriers by upregulating cytokines and chemokines via the Tolllike receptor. The major pro-inflammatory factors, including $M y d 88, N F-\kappa B$, $1 / 6$, and II8, were upregulated compared to controls. After being challenged with $E$. coli, the survival rate of chickens fed with LGG was significantly higher than those in the control group, and decreased numbers of $E$. coli were detected in the heart and lungs of the LGG group. In summary, oral administration of LGG to chickens could improve growth performance, maintain intestinal homeostasis, and enhance innate immune response and disease resistance.

Keywords: adhesion, intestinal microbiota, innate immune response, disease resistance, Lacticaseibacillus rhamnosus

\section{INTRODUCTION}

Administration of antibiotics is the most common treatment of bacterial diseases on livestock farms. Despite the improvement in growth performance, antibiotics have caused a number of problems (Simoneit et al., 2014). The administration of antibiotics can disrupt the balance of intestinal flora (Rashid et al., 2012), while excessive and unreasonable usage of antibiotics can generate drug-resistant bacteria (Lupindu et al., 2015). Therefore, a safe alternative to antibiotics is urgently needed. A dietary supplement of probiotics could improve growth performance, regulate immune response, prevent disease, and avoid a rise in drug resistance (Ng et al., 2009). 
In addition, administration of probiotics contributes to balancing intestinal microflora and reducing the oxygen in the intestines, which is essential for some pathogens (Pradhan et al., 2020). The intestinal microflora is closely correlated with the growth performance and health of the host; it plays an important role in regulating physiological function (Stanley et al., 2012) and can stimulate the development of intestinal cells, promote the absorption of nutrients, and regulate immunity (Abrams et al., 1963). Probiotics can improve immunity by influencing the intestinal microflora of chickens.

Probiotics like Lactobacillus and Bacillus subtilis have been widely used to promote immunity and prevent diseases in scientific research and clinical applications. The Lacticaseibacillus rhamnosus GG (LGG) strain can produce short-chain fatty acids, promoting intestinal health and improving immunity. LGG has been shown to improve growth performance, treat and prevent diarrhea, and act as an antioxidant (Wang et al., 2019). It is successfully used to prevent and treat human diarrhea, dental caries, and other diseases. Recently, it was confirmed that the metabolites of LGG promote the formation of intestinal mechanical barriers and immune barriers in newborn rats, as well as enhancing resistance to Escherichia coli (E. coli) infections (He et al., 2017). Moreover, LGG can produce 92 kinds of proteins under acidic conditions, most of which are related to forming biofilms, maintaining the structure of cell membranes, and regulating immune responses (Savijoki et al., 2011). Among these, the SpaC protein is an essential protein for LGG to adhere to the intestinal mucosa and to induce epithelial cells that produce reactive oxygen species (Yan et al., 2013). The novel protein HM0539 shows a potent protective effect on the intestinal barrier (Gao et al., 2019).

Innate immunity is the first line of defense against the colonization of pathogens, and it plays a critical role in the pathogenesis and progression of intestinal disorders. Previous research has shown that administration of probiotics could upregulate the expressions of Toll-like receptors (TLRs) and activate an innate immune response (Grabig et al., 2006). To gain better insight into the role of LGG on chickens, the probiotic effects of LGG were assessed. The adhesion ability and inhibitory effects on pathogenic bacteria were evaluated in vitro. The growth performance, the intestinal homeostasis, the immune response, and the disease resistance were explored in chickens.

\section{MATERIALS AND METHODS}

\section{Bacteria}

Lacticaseibacillus rhamnosus GG (ATCC 53103) was obtained from the American Type Culture Collection and was cultured in MRS broth at $37^{\circ} \mathrm{C}$. The bacterial pathogens of Staphylococcus aureus (ATCC 25923), Salmonella Paratyphi B (CMCC 50094), and Salmonella Enteritidis (CICC 24119) were stored in our laboratory and cultured in $\mathrm{LB}$ broth at $37^{\circ} \mathrm{C}$. O 1 E. coli was isolated from clinically infected ducks suffering from colibacillosis and stored in our laboratory.

\section{Primary Culture of Chicken Intestinal Epithelial Cells}

Primary duodenal intestinal epithelial cells were prepared from 19-day-old specific pathogen-free chicken embryos as described previously (Guo et al., 2015; Zhang et al., 2019). The embryos were dissected, and the duodenal intestines were transferred to Hank's Balanced Salt Solution (HBSS) supplemented with $100 \mathrm{U} / \mathrm{mL}$ of penicillin and streptomycin. Then the duodenal intestines were cut into small pieces and washed three times with HBSS. Thereafter, the duodenal intestines were digested with collagenase I $(1 \mathrm{mg} / \mathrm{mL})$ for $50 \mathrm{~min}$ at $37^{\circ} \mathrm{C}$ under steady agitation. The cell pellets were centrifuged at $800 \mathrm{rpm}$ for $10 \mathrm{~min}$ and washed twice with HBSS. The cell pellets were resuspended in DMEM/F12 medium supplemented with epidermal growth factor $(20 \mathrm{ng} / \mathrm{mL})$, heparin sodium salt $(100 \mu \mathrm{g} / \mathrm{mL})$, insulin (5 $\mu \mathrm{g} / \mathrm{mL})$, and $2.5 \%$ fetal bovine serum. The larger pieces were filtered with a 200-mesh sieve. Fibroblasts and macrophages were discarded by $2 \mathrm{~h}$ adherence. Then, non-adherent cells were transferred to a new dish and incubated for $48 \mathrm{~h}$.

\section{Screening for Probiotic Properties in vitro}

\section{Antimicrobial Activities}

The antibacterial experiment followed the method described by Zhang D. et al. (2012) and Hwanhlem et al. (2017). The pathogenic bacteria (S. aureus, S. Paratyphi B, S. Enteritidis, and

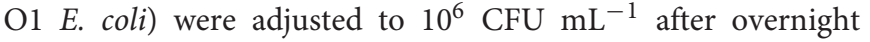
cultivation. The pathogenic bacteria were spread onto the LB agar plates. The LGG culture $\left(\mathrm{OD}_{600}=1.0\right)$ was centrifuged at 3,000 rpm for $10 \mathrm{~min}$ to obtain the culture supernatant of LGG. After centrifugation, the LGG cells were washed twice with PBS and resuspended. In addition, the LGG culture $\left(\mathrm{OD}_{600}=1.0\right)$ was frozen and thawed twice, then ultrasonically broken, and centrifuged at 3,000 rpm for $10 \mathrm{~min}$ to obtain the lysate. The LGG culture, lysate, culture supernatant, and bacterial cells were prepared and transferred to holes (approximately $5 \mathrm{~mm}$ in diameter) punched into the LB plates. Then, the LB agar plates were incubated at $37^{\circ} \mathrm{C}$ for $24 \mathrm{~h}$. The antibacterial activities were determined by the size of the inhibition zone. Three replicates per sample were performed.

\section{LGG Adhesion}

Investigation of the adhesion of the LGG to primary chicken intestinal epithelial cells followed the method described by Fernández de Palencia et al. (2008). The LGG and E. coli cells were added to chicken intestinal epithelial cells $(\mathrm{MOI}=100: 1)$; then, the cells were incubated at $37^{\circ} \mathrm{C}$ and $5 \% \mathrm{CO}_{2}$ for $1 \mathrm{~h}$. Unbound bacteria were softly washed away with PBS; then, the cells were lyzed with $1 \%$ Triton X-100 for $20 \mathrm{~min}$ and spread onto MRS agar plates for a viable count. Three replicates per sample were performed.

To test the competition between LGG and E. coli for cell adhesion, LGG and E. coli (1:1) were simultaneously added to chicken intestinal epithelial cells and incubated for $1 \mathrm{~h}$. Unbound bacteria were removed, and E. coli counts were carried out. The ability of LGG to inhibit the adhesion of E. coli was assessed 
TABLE 1 | Composition and nutrient levels of basal diets (air-dry basis).

\begin{tabular}{lccc}
\hline Ingredients & Content & Nutrient levels & Content \\
\hline Corn & 50.75 & $\mathrm{ME} /(\mathrm{MJ} / \mathrm{kg})$ & 11.3 \\
Soybean meal & 32.94 & $\mathrm{CP}$ & 19.06 \\
Fish meal & 2.01 & $\mathrm{Met}$ & 0.50 \\
$\mathrm{CaHPO}_{4}$ & 1.92 & $\mathrm{Lys}$ & 1.15 \\
Limestone & 1.25 & $\mathrm{Ca}$ & 1.00 \\
Corn protein flour & 4.83 & & \\
NaCl & 0.30 & & \\
Wheat & 5.00 & & \\
Premix & 1 & & \\
Total & 100 & &
\end{tabular}

Per kg of premix: Vitamin A, 10,000 U; Vitamin $B_{1}, 6.0$ mg; Vitamin $B_{12}, 40.0$ mg; Vitamin $D_{3}, 7,000 \mathrm{U}$; Vitamin E, $50 \mathrm{U}$; Vitamin $B_{7}, 2.0 \mathrm{mg}$; Vitamin $B_{3}, 32.5 \mathrm{mg}$; Cu, 8.0 mg; Fe, 90.0 mg; Mn, 70.0 mg; Zn, 90.0 mg; I, 1.1 mg; Se, 0.2 mg.

as follows: LGG was first added to intestinal epithelial cells and incubated for $1 \mathrm{~h}$, after which unbound LGG was removed, and $E$. coli was added to the wells. After incubation for $1 \mathrm{~h}$, adherent $E$. coli was counted. To test the ability of LGG to displace previously adhered E. coli, E. coli was first added to intestinal epithelial cells and incubated for $1 \mathrm{~h}$, after which unbound $E$. coli was removed and LGG was added to the wells. After incubation for $1 \mathrm{~h}$, adherent $E$. coli was counted.

\section{Animal Experiments}

Healthy newborn Ross 308 chickens were raised under the same conditions with sufficient water and food, and randomly allotted to two groups (basal diet or supplemented with $10^{6} \mathrm{CFU} \mathrm{g} \mathrm{g}^{-1}$ LGG). The composition and nutrient levels of the basal diets are shown in Table 1. Each group consisted of three replicates with 30 chickens per replicate. The chickens were weighed individually, and blood samples were collected from the wing vein at days 14 and 21. On day 21, five chickens per group were randomly selected and euthanized. The fecal contents, blood, spleens, and livers were collected. IgG, IgM, and $\operatorname{sgA}$ in serum were detected by using chicken Immunoglobulin $G$ ELISA kit, chicken Immunoglobulin M ELISA kit, and chicken secretory Immunoglobulin A ELISA kit (SenBeiJia, Nanjing, China), respectively. The remaining chickens were challenged with $10^{6} \mathrm{CFU}$ E. coli. Five chickens per group were randomly selected and euthanized at 1 and 3 days post-infection (dpi). The hearts, livers, spleens, lungs, and kidneys were collected.

All experiments were carried out in accordance with the principles of the Basal Declaration and Recommendations of Committee on the Ethics of Animal Experiments of Yangzhou University, and the protocol was approved by the Committee on the Ethics of Animal Experiments of Yangzhou University.

\section{S rRNA Sequencing and Analysis}

The DNA in the fecal content was extracted using HiPure Stool DNA Kit B (Magen, Shanghai, China), according to the manufacturer's protocols. The highly variable regions of V3 and V4 on 16S rRNA were amplified using 20-30 ng DNA as templates (F: CCTACGGRRBGCASCAGKVRVGAAT; R: GGACTACNVGGGTWTCTAATCC). Illumina MiSeq/NovaSeq was used for two-terminal sequencing; the sequenced reads were
TABLE 2 | Primers used in this study.

\begin{tabular}{|c|c|}
\hline Primer name & Sequence $\left(5^{\prime}-3^{\prime}\right)$ \\
\hline Mhc Il $\alpha-F$ & TGGGATCCTCCGTCCTGAAGCCGCAC \\
\hline Mhc $/ / \alpha-R$ & GCGTCGACTCAGAGCAGCCCCGGTT \\
\hline Myd88-F & TGATGCCTTCATCTGCTACTG \\
\hline Myd88-R & TCCCTCCGACACCTTCTTTCTA \\
\hline$N F-\kappa b-F$ & CAGCCCATCTATGACAACCG \\
\hline$N F-$ кb-R & TCCCTGCGTCTCCTCTGTGA \\
\hline$\| 1 \beta-F$ & GTGAGGCTCAACATTGCGCTGTA \\
\hline$\| 1 \beta-R$ & TGTCCAGGCGGTAGAAGATGAAG \\
\hline$\| 8-\mathrm{F}$ & ATGAACGGCAAGCTTGGAGCTG \\
\hline$\| 8-\mathrm{R}$ & TCCAAGCACACCTCTCTTCCATCC \\
\hline Ifn- $\alpha-F$ & ATGCCACCTTCTCTCACGAC \\
\hline Ifn- $\alpha-R$ & AGGCGCTGTAATCGTTGTCT \\
\hline $1 / 6-\mathrm{F}$ & TCTGTTCGCCTTCAGACCTA \\
\hline $116-\mathrm{R}$ & GACCACCTCATCGGGATTTAT \\
\hline TIr4-F & AGTCTGAAATTGCTGAGCTCAAAT \\
\hline TIr4-R & GCGACGTTAAGCCATGGAAG \\
\hline$\beta$-actin-F & GAGAAATTGTGCGTGACATCA \\
\hline$\beta$-actin-R & ССТGAАССТСТСАТTGCСА \\
\hline
\end{tabular}

spliced and filtered, and the chimera was removed. Obtained sequences were performed with operational taxonomic units (OTUs) clustering and diversity analysis.

\section{Quantitative Real-Time PCR}

Total RNA was extracted from the spleens and livers using TRIzon Reagent (CoWin Biosciences, Beijing, China), and $1 \mu \mathrm{g}$ of the total RNA was reverse-transcribed according to the instructions of the TranScript all-in-one first-strand cDNA Synthesis Supermix kit (Transgen, Beijing, China). The TransStartR Tip Green qPCR SuperMix kit (Transgen, Beijing, China) was used for qRT-PCR. The primer sequences used for qRT-PCR are listed in Table 2. The qRT-PCR was conducted on a total volume of $20 \mu \mathrm{L}$, and the amplification steps consisted of $94^{\circ} \mathrm{C}$ for $30 \mathrm{~s}, 40$ cycles of denaturation at $94^{\circ} \mathrm{C}$ for $5 \mathrm{~s}$, and extension $60^{\circ} \mathrm{C}$ for $34 \mathrm{~s}$, as well as a dissociation curve analysis. The $2^{-\Delta \Delta C T}$ method was used to estimate mRNA abundance. Relative gene expression levels were normalized by the housekeeping gene $\beta$-actin.

\section{Statistical Analysis}

The Student's $t$-test was used to identify significant differences between experimental groups using the SPSS computer software (SPSS, Chicago, IL, United States). A $p$-value of $<0.05$ was considered the threshold for statistical significance.

\section{RESULTS}

\section{Antibacterial Activity of LGG}

To investigate the antimicrobial activities of LGG, the LGG culture, culture supernatant, lysate, and cells were prepared. The LGG culture, culture supernatant, and lysate showed good inhibitory ability against the growth of E. coli, S. aureus, S. Paratyphi B, and $S$. Enteritidis, and the inhibitory zone was higher 

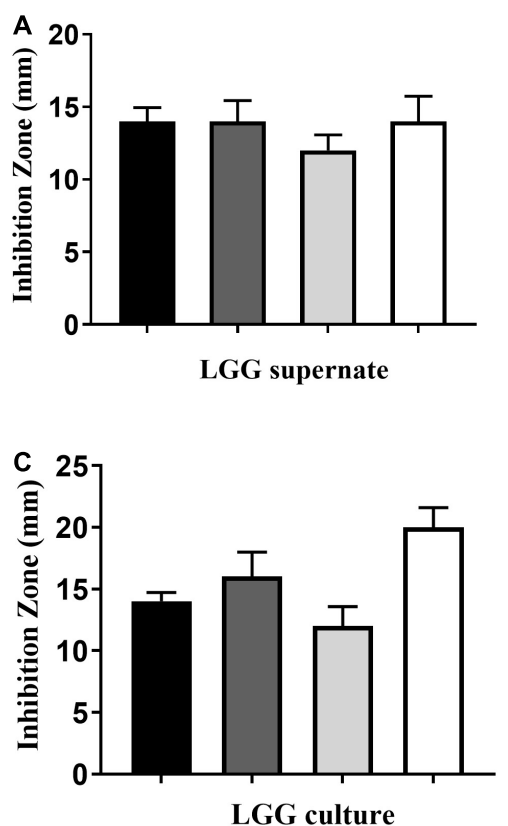
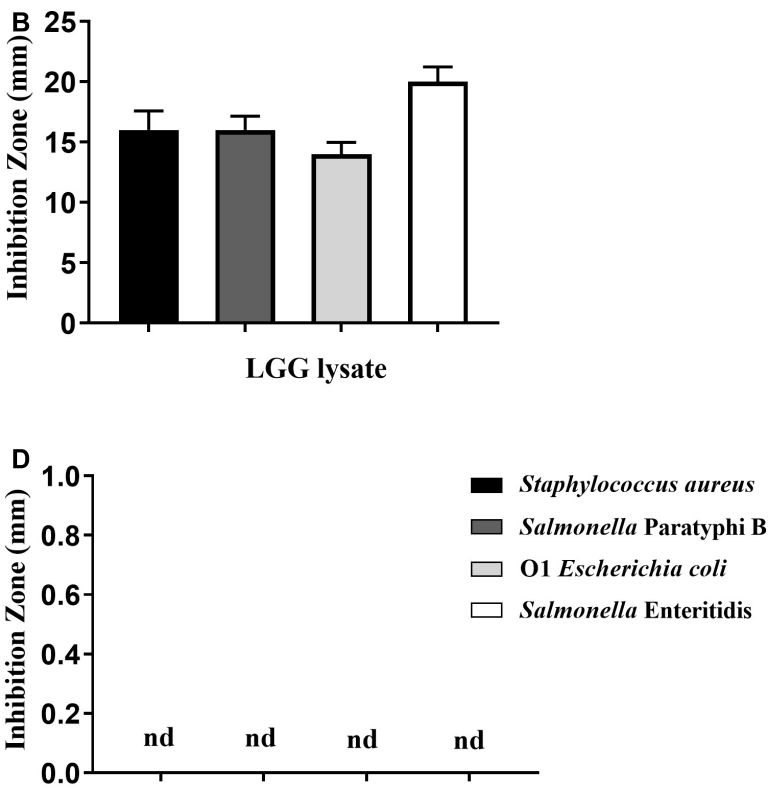

LGG cells

FIGURE 1 | Antibacterial activity of LGG against Staphylococcus aureus, Salmonella Paratyphi B, O1 E. coli, and Salmonella Enteritidis. Four pathogenic bacteria were spread onto the LB agar plates. Then (A) LGG culture supernatant, (B) lysate, (C) LGG culture, and (D) LGG cells were transferred to holes (5-mm diameter) punched into the agar palates. The antimicrobial activity was determined by the size of the inhibition zone. Bars represent the means \pm standard deviations of three independent repetitions. $\mathrm{Nd}$, not detected.
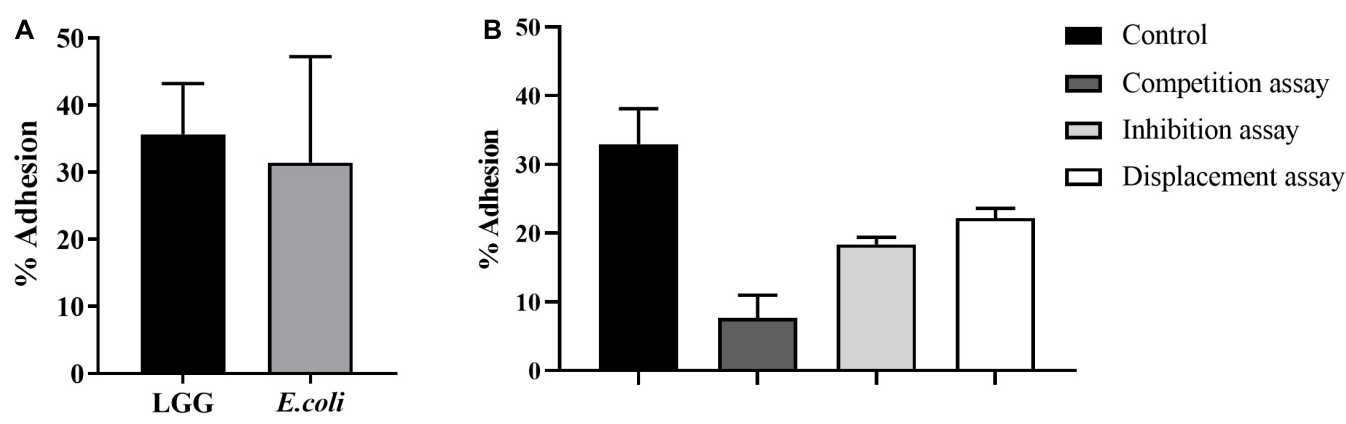

FIGURE 2 | Effects of LGG on E. coli adhesion to primary chicken intestinal epithelial cells. (A) The LGG and E. coli cultures were added to primary chicken intestinal epithelial cells $(\mathrm{MOI}=100: 1)$, followed by 1 -h incubation at $37^{\circ} \mathrm{C}$ and $5 \% \mathrm{CO}_{2}$. Adhesion percentage of LGG and $\mathrm{E}$. coli to primary chicken intestinal epithelial cells was expressed as the number of adherent bacteria relative to the total number of bacteria. (B) Competition, inhibition, and displacement of $E$. coli adhesion in the presence of LGG. LGG and E. coli (1:1) were simultaneously added to primary chicken intestinal epithelial cells for competition assay. In the inhibition assay, LGG was first added to primary chicken intestinal epithelial cells and incubated for $1 \mathrm{~h}$, after which unbound LGG was removed and E. coli was added to the wells. On the contrary, in the displacement assay, E. coli was added first. Bars represent the means \pm standard deviations of three independent repetitions.

than $12 \mathrm{~mm}$ (Figures 1A-C). Of these pathogens, the inhibitory effect on Salmonella Enteritidis was the best. However, LGG cells could not inhibit the pathogenic bacteria (Figure 1D).

\section{Effects of LGG on E. coli Adhesion to Primary Chicken Intestinal Epithelial Cells}

One of the important criteria for probiotics is the ability to adhere to the intestinal mucosa. The adhesion levels of LGG and E. coli to chicken intestinal epithelial cells were 35.3 and $31.5 \%$, respectively (Figure 2A). In the competition assay, LGG showed the best performance in preventing $E$. coli adhesion to intestinal epithelial cells, as it reduced $75.7 \%$ of the E. coli adhesion. In the inhibition assay, the E. coli adhesion was reduced by $41.7 \%$. In the displacement assay, the adhesion levels of E. coli were $22.2 \%$ (Figure 2B).

\section{LGG Promotes Growth Performance and Immunoglobulin}

As shown in Table 3, compared to the control group, chickens fed with LGG had significantly increased body weight $(p<0.05)$. On 
TABLE 3 | Effects of dietary LGG on body weight and immunoglobulins in chickens.

\begin{tabular}{|c|c|c|}
\hline & Control & LGG \\
\hline \multicolumn{3}{|c|}{ Average weight gain (g/day) } \\
\hline 14 days & $16.4 \pm 2.9^{a}$ & $19.0 \pm 2.6^{b}$ \\
\hline 21 days & $31.2 \pm 3.6^{a}$ & $33.9 \pm 3.1^{b}$ \\
\hline \multicolumn{3}{|c|}{ Concentration of Immunoglobulins, $\mu \mathrm{g} / \mathrm{mL}$} \\
\hline slgA (14 days) & $78.8 \pm 7.4^{\mathrm{a}}$ & $97.0 \pm 6.9^{b}$ \\
\hline $\operatorname{lgG}$ (14 days) & $429.9 \pm 74.7$ & $534.5 \pm 47.7$ \\
\hline IgM (14 days) & $56.9 \pm 6.1$ & $54.1 \pm 4.3$ \\
\hline slgA (21 days) & $68.5 \pm 11.0^{a}$ & $84.4 \pm 12.4^{b}$ \\
\hline $\operatorname{lgG}$ (21 days) & $503.0 \pm 57.0^{\mathrm{a}}$ & $726.7 \pm 80.1^{\mathrm{b}}$ \\
\hline $\lg \mathrm{M}$ (21 days) & $38.4 \pm 9.1^{\mathrm{a}}$ & $58.1 \pm 5.9^{b}$ \\
\hline
\end{tabular}

Data are expressed as means \pm standard deviations $(n=5)$.

$a, b$ Values in the same row with different superscripts differ significantly $(p<0.05)$.

day 21, the average weight of chickens in the experimental group was $423.7 \pm 56.6 \mathrm{~g}$, while the average weight of chickens in the control group was $397 \pm 54.4 \mathrm{~g}$.

Chickens fed with a diet of $10^{6} \mathrm{CFU} \mathrm{g}^{-1} \mathrm{LGG}$ had higher IgM, IgG, and sIgA than chickens in the control group. On day 21 , the serum concentrations of $\operatorname{IgA}, \operatorname{IgG}$, and $\operatorname{IgM}$ in the experimental group were $84.4 \pm 12.4,726.7 \pm 80.1$, and $58.1 \pm 5.9 \mathrm{~g} / \mathrm{mL}$, respectively, which were significantly higher than the controls $(p<0.05)$.

\section{Taxonomic Composition of Intestine Microbiota}

The 16S rRNA sequence analysis was conducted after 21 days of feeding LGG. The 16S rRNA sequences have been deposited in the Sequence Read Archive (PRJNA699761). As shown in Figure 3A, the OTUs rank abundance indicated that adequate sequence coverage was obtained to reflect the diversity of samples. The species richness of the LGG group was higher than that of the control group. The number of OTU species in the LGG group was 170-more than that in the control group (Figure 3B). The principal component analysis (PCA) and principal coordinates analysis (PCoA) plots showed a good separation of intestine microbiota between the LGG group and the control group (Figures $3 \mathbf{C , D}$ ). These results indicated that being fed with LGG could increase the diversity of cecum microbiota.

As shown in Figure $\mathbf{4 A}$, the main phyla were Firmicutes, Cyanobacteria, and Proteobacteria. After 21 days of LGG feed, the higher percentages at the family level were Ruminococcaceae (56.03\%), Lachnospiraceae (24.98\%), Lactobacillaceae (10.12\%), and the Clostridiales vadinBB60 group (4.8\%) (Figure 4B). The relative abundance of Ruminococcaceae and Lactobacillus in chickens fed with a diet of LGG was higher than that in the control group (Figures $4 \mathbf{C , E}$ ). In contrast, the relative abundance of Lachnospiraceae in chickens fed with a diet of LGG was significantly lower than that in the control group $(p<0.05$, Figure 4D). The metastats difference analysis showed that the largest differences between the LGG group and the control group were Butyricicoccus, DTU089, GCA900066575, Lactobacillus, and Ruminococcaceae_UCG-013. The levels of DTU089, Lactobacillus, and Ruminococcaceae_UCG-013 in chickens fed with LGG were $0.20,0.12$, and $1.07 \%$, which were all higher than those of the control group. The relative abundance of Butyricicoccus and GCA-900066575 in chickens fed with a diet of LGG was significantly lower than that of the control group (Figure 4F).

\section{The Expression of Innate Immune-Related Genes Induced by LGG}

To investigate the response of innate immunity in chickens fed with a diet of LGG, the innate immune-related genes were detected in the spleens and livers of chickens fed with LGG compared to the controls after 21 days of feeding. As shown in Figure 5, LGG-supplemented diets upregulated the expressions of innate immune-related genes in the spleens, especially Mhc II$\alpha, I l 6$, and Il8. However, this induction of innate immune-related genes does not show an obvious change in the liver.

\section{Survival Rate and E. coli Content}

The survival rate of chickens fed with LGG was higher than that of controls after E. coli infection. None of the chickens in the LGG group died by $3 \mathrm{dpi}$, while the chickens in the control group continued to die until $6 \mathrm{dpi}$ (Figure 6A). The number of E. coli was significantly lower in the hearts and lungs of chickens fed with a diet of LGG than in those of the control group at 1 and 3 dpi. However, the E. coli content in the livers and spleens showed no difference between the two groups. The E. coli content in the kidneys was significantly lower in the experimental group than the control group at $3 \mathrm{dpi}$ (Figure 6B).

\section{Innate Immune-Related Genes in the Spleen and Liver of the Infected Chickens}

After being challenged with E. coli, the innate immune response was significantly activated in the spleens of chickens fed with LGG compared to the controls. The expressions of Tlr4 and Mhc $I I-\alpha$ were upregulated by 3.6- to 25.6-fold in the spleen at 1 and 3 dpi (Figures 7A,B). In turn, downstream signal transducing adaptor protein $M y d 88$ and signaling molecule $N F-\kappa B$ were also activated in the spleen at 1 and $3 \mathrm{dpi}$ (Figures 7C,D). Moreover, the expressions of proinflammatory cytokines (Ifn- $\alpha, I l 1 \beta$, and Il6) were significantly upregulated in the spleen in LGG-fed chickens compared to controls at 1 and 3 dpi (Figures 7E-G). The expressions of $I l 8$ were significantly upregulated in the spleen and the liver at 1 dpi but were significantly downregulated by about 0.2 -fold at $3 \mathrm{dpi}$ (Figure $7 \mathbf{H}$ ).

\section{DISCUSSION}

The important criterion for probiotics is their ability to adhere to intestinal mucosa. Adhesion of Lactobacillus to epithelial cells occurs mainly through adhesins, including lipoteichoic acid, S-layer protein, and peptidoglycans (Lönnermark et al., 2012). In 
A

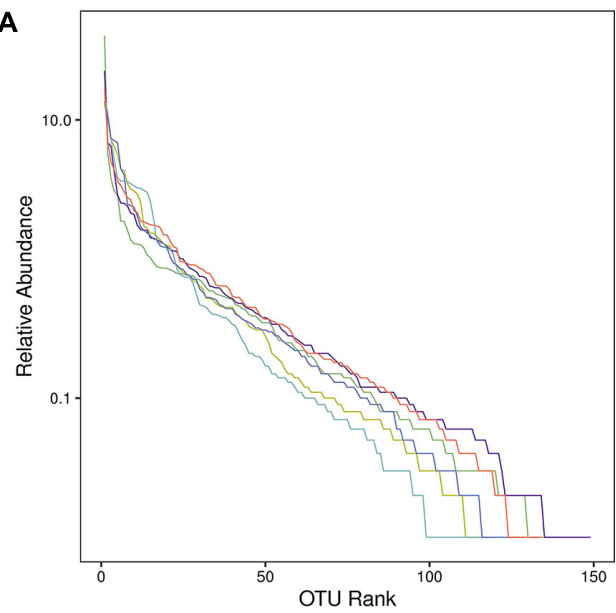

C

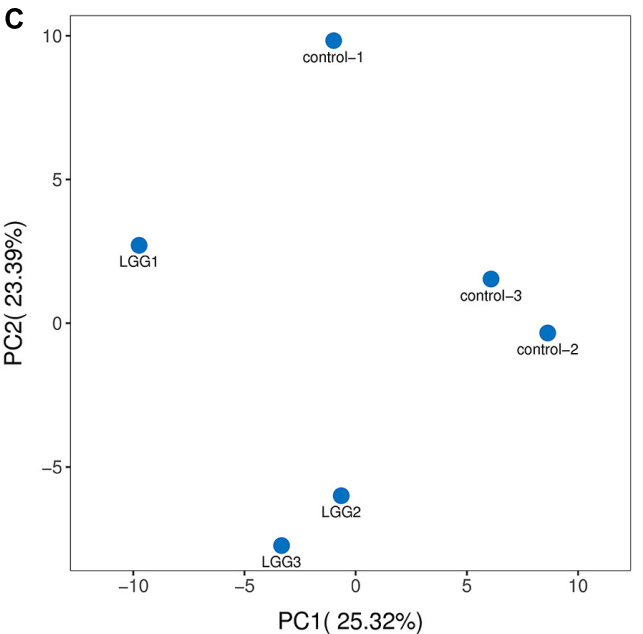

B

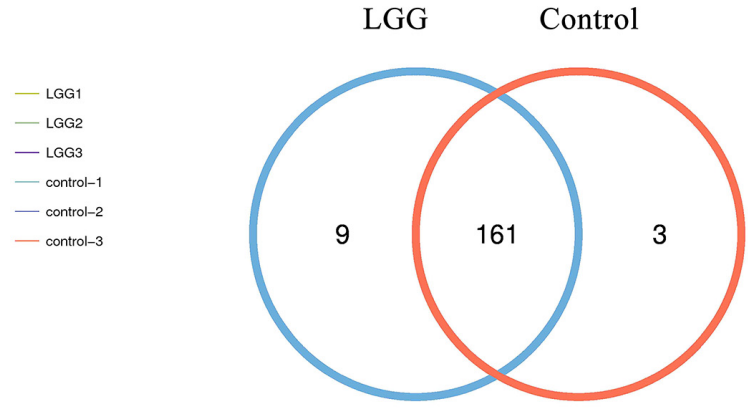

D

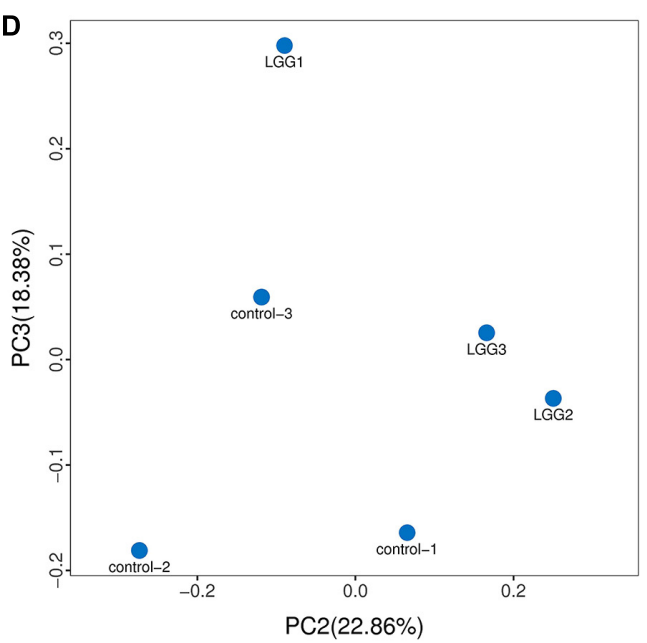

FIGURE 3 | Effects of dietary LGG on cecal microbial diversity in chickens. The cecal contents of chickens fed with LGG and the controls were collected for high-throughput sequence analysis after 21 days of feeding. (A) $\alpha$-diversity of OTU rank abundance. (B) OTU Venn diagram between the LGG group and the control group. (C) Principal component analysis (PCA) plot based on the distribution of bacterial community. (D) Principal coordinates analysis (PCoA) plot using Brary-Curtis distances. The percentage represents the contribution of the principal component to the sample difference.

previous reports, LGG was demonstrated to be able to adhere to the intestines of infants and adults (Kirjavainen et al., 1998) and to inhibit the adhesion of E. coli K88 and Salmonella typhimurium to Caco-2 cells (Bogovic Matijasić et al., 2006). Similar to previous research, our results showed that LGG demonstrates good adhesion to primary chicken intestinal epithelial cells. Meanwhile, LGG could reduce the adhesion of $E$. coli to primary chicken intestinal epithelial cells through competition, inhibition, and displacement. Probiotics adhere to and colonize in the intestinal mucous membrane to form a pellicle barrier, which prevents the adhesion of pathogenic bacteria (Fernández et al., 2003; Bogovic Matijasić et al., 2006). L. rhamnosus can produce bacteriocin, organic acid, and hydrogen peroxide, which can inhibit the proliferation of pathogens. A previous study showed that L. rhamnosus could inhibit the proliferation of E. coli, Salmonella, and Clostridium perfringens (Abhisingha et al., 2018). In this study, LGG cultures, culture supernatant, and lysate showed strong inhibitory effects on S. aureus, E. coli, S. Paratyphi B, and S. Enteritidis. Moreover, the inhibition zones of cultures and lysate are 1-5 $\mathrm{mm}$ higher than that of the culture supernatant. Based on the above findings, LGG can colonize in the intestinal tract and inhibit the proliferation of some pathogenic bacteria.

It has previously been reported that LGG can promote the weight of weaned pigs (Bocourt et al., 2004). In this study, chickens fed with LGG exhibited a greater average daily weight gain. In another study, dairy infused with LGG and Bacillus licheniformis could promote laying rates, egg production, and average weights of eggs (Zhang J. L. et al., 2012). Moreover, a high-throughput sequence analysis of bacterial 16S rRNA showed that feeding chickens with LGG increased the diversity of intestinal microbiota. In chickens fed with LGG for 21 days, the relative abundance of Lactobacillus was significantly higher than that in the control group. The lactic acid produced by 

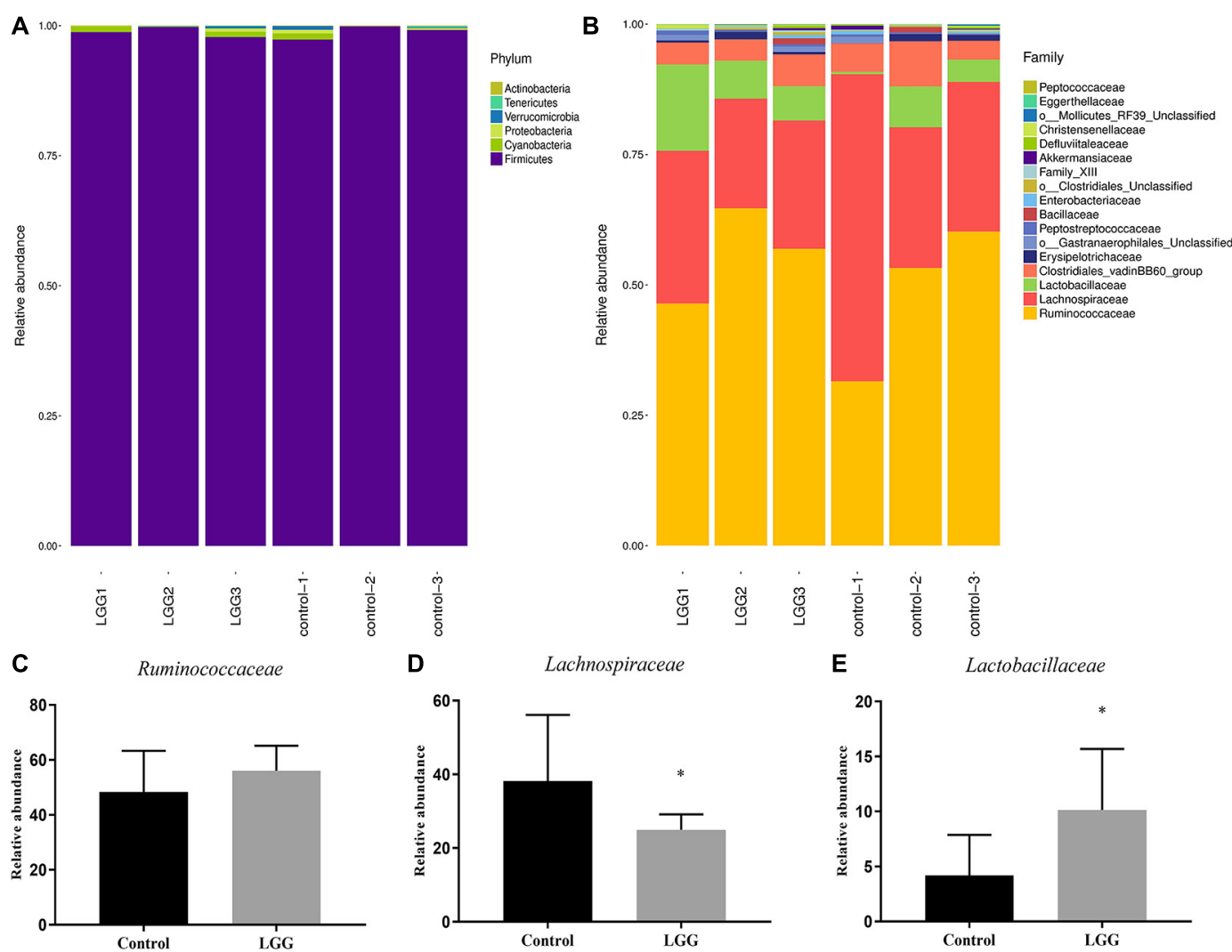

E Lactobacillaceae
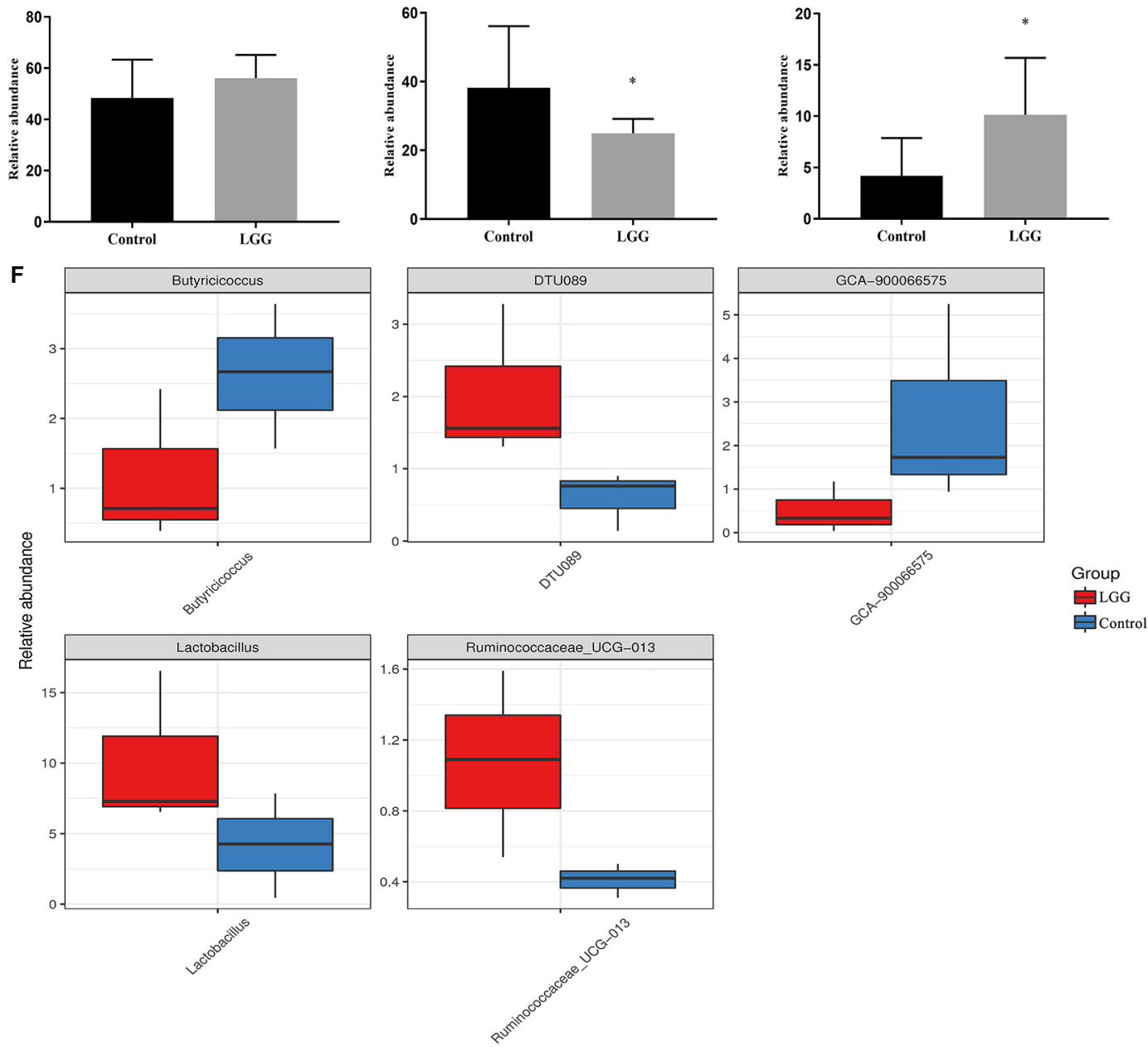

FIGURE 4 | Comparison of identified relative abundance in cecal microbes. Relative abundance of bacterial (A) phylum level and (B) family level. Relative abundance of (C) Ruminococcaceae, (D) Lachnospiraceae, and (E) Lactobacillaceae. Bars represent the means \pm standard deviations of three independent repetitions. ${ }^{*} p<0.05$. (F) The differential abundance between LGG and the control group based on metastats difference analysis. The abundance distribution of the five species with the greatest difference between the two groups was presented. 

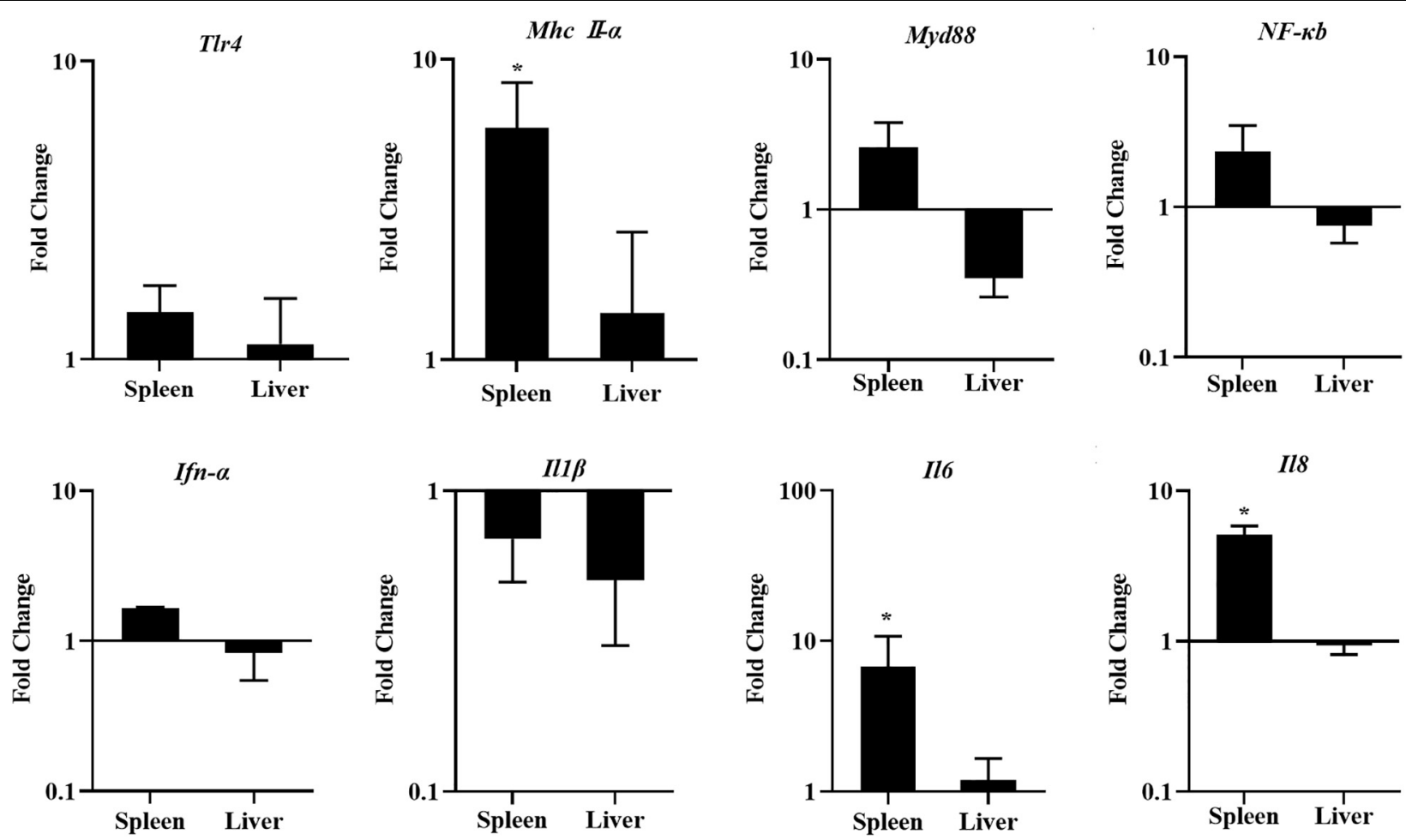

FIGURE 5 The expression of T/r4, Mhc II- $\alpha, M y d 88, N F-\kappa B$, Ifn- $\alpha, \| 1 \beta$, I/6, and //8 in the spleens and livers of chickens after 21 days of feeding. The fold change represents the target gene expression in the diet with LGG compared to the diet of the control group. The relative gene expression levels were normalized to $\beta$-actin. Bars represent the means \pm standard deviations $(n=5) .{ }^{*} p<0.05$.
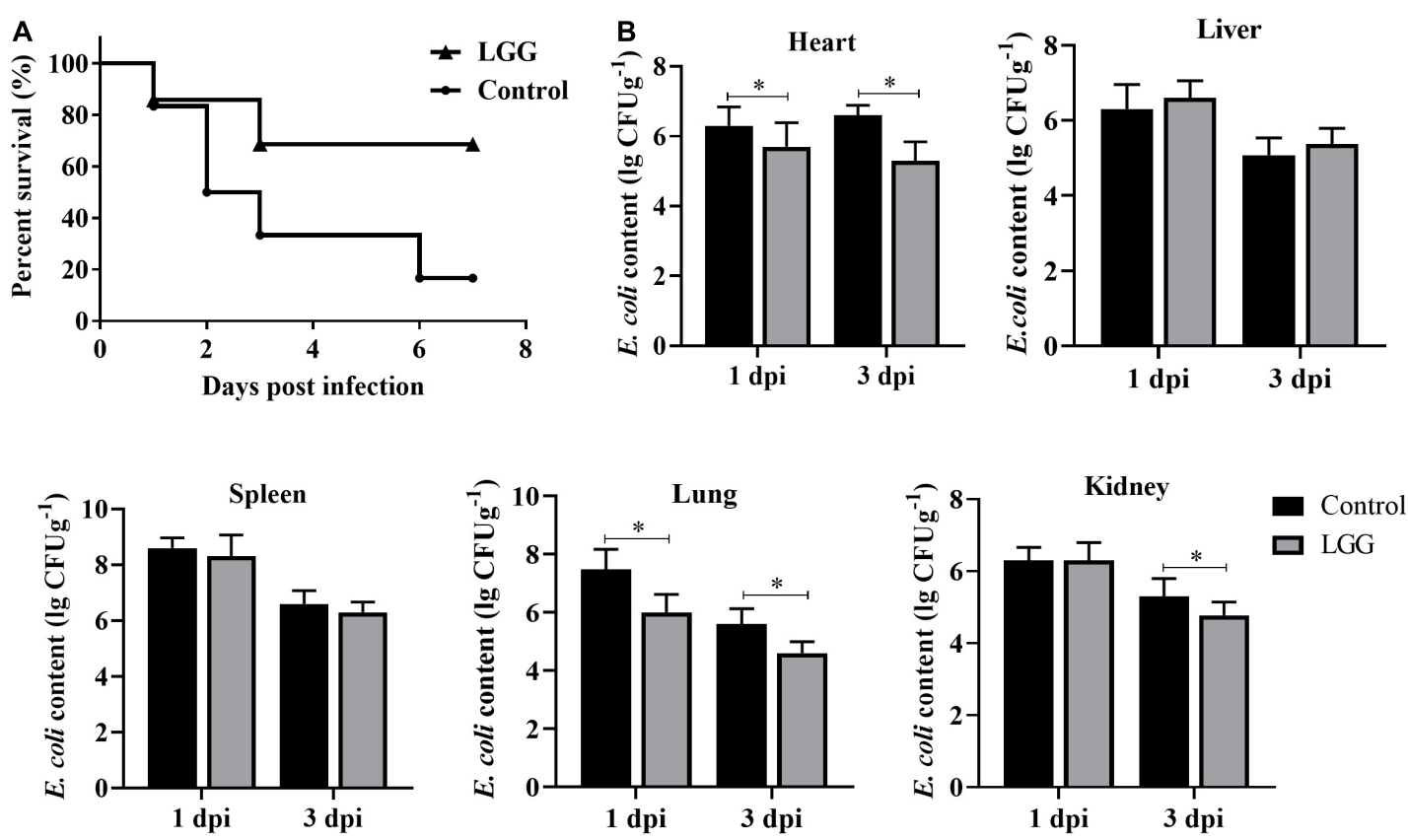

FIGURE 6 | Disease resistance of chickens fed with LGG diet post-challenge with E. coli. Chickens were challenged with $10^{6}$ CFU E. coli after 21 days of feeding. (A) The survival rate of chickens after infection with E. coli. (B) E. coli content in hearts, livers, spleens, lungs, and kidneys of infected chickens at 1 and 3 dpi (log 10 CFU $\left.g^{-1}\right)$. Bars represent the means \pm standard deviations $(n=5) .{ }^{*} p<0.05$. 

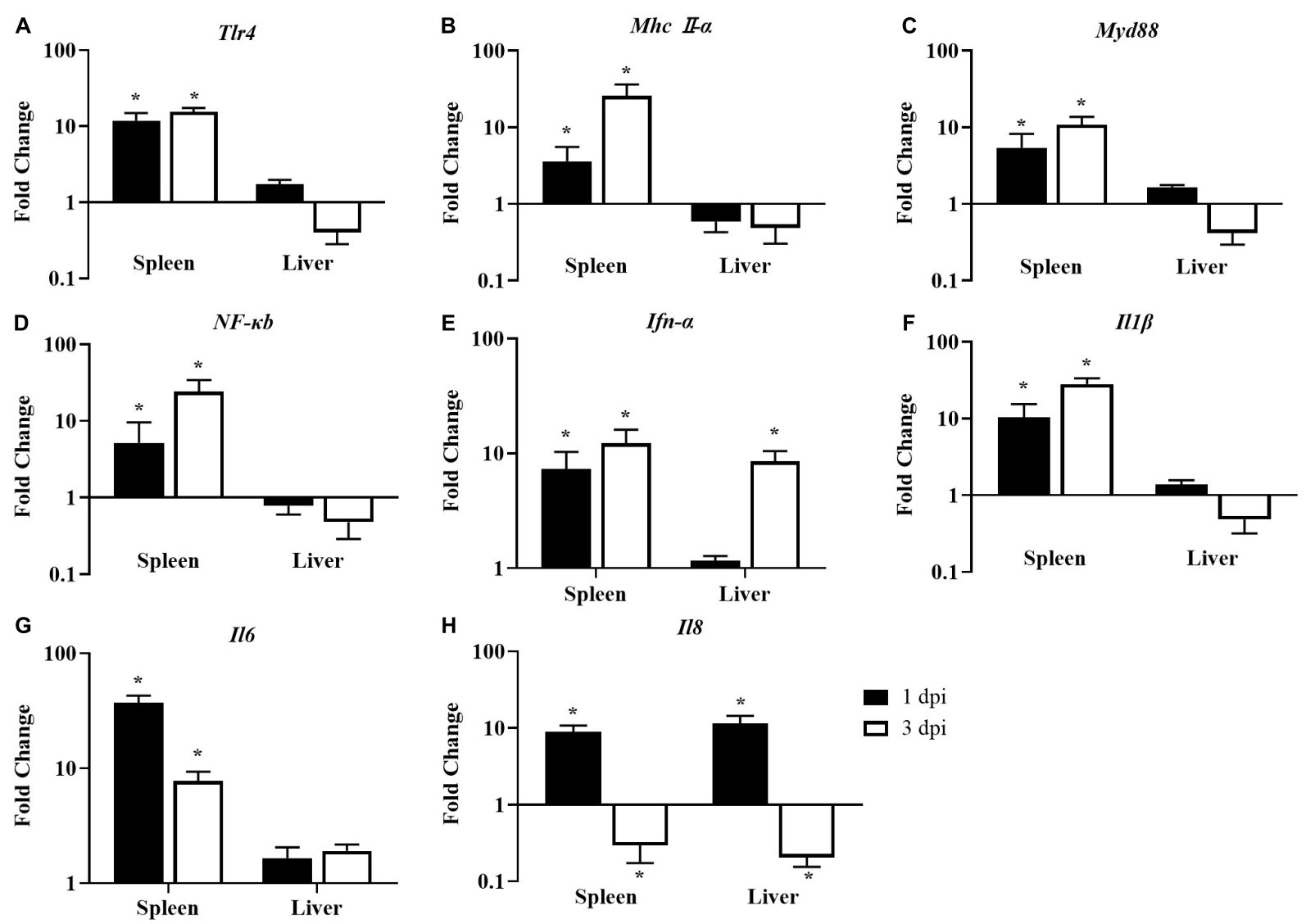

FIGURE 7 | The expression of immune-related genes in the spleens and livers of infected chickens at 1 and 3 dpi. Expression of (A) T/r4, (B) Mhc II- $\alpha$, (C) Myd88, (D) $N F-\kappa B$, (E) Ifn- $\alpha,(\mathbf{F}) / 1 \beta$, (G) $/ / 6$, and (H) //8. The fold change represents the target gene expression in the diet with LGG compared to the diet of the control group. The relative gene expression levels were normalized to $\beta$-actin. Bars represent the means \pm standard deviations $(n=5)$. ${ }^{\star} p<0.05$.

Lactobacillus can inhibit the growth of some pathogenic bacteria (Wang et al., 2019). At the same time, the level of Clostridium in chickens fed with LGG was lower. Clostridium difficile can produce a variety of exotoxins that have a negative effect on animals (Aschfalk and Müller, 2002).

In innate immunity, the intestinal mucosa is considered to be the first line of defense against pathogen infection. A previous study found less mucus on the surfaces of intestinal mucosa in chickens with less intestinal flora, indicating that intestinal microflora may be involved in establishing the mucosal layer (Forder et al., 2007). Our results indicated that a diet with LGG can regulate intestinal microbiota and maintain intestinal health. In return, the increase in intestinal microbiota diversity and intestinal health can improve growth performance and immune ability (Pereira et al., 2019). In a previous report, LGG was found to decrease the rate of diarrhea in weaned pigs infected with E. coli and to upregulate the concentrations of sIgA in the jejunum and ileum (Zhang et al., 2010). Similarly, in this study, a diet with LGG significantly upregulated the concentrations of the immunoglobulins sIgA, IgG, and IgM.

Previous research demonstrated that a diet with probiotics could upregulate the expressions of Tlr2, Tlr3, Tlr4, Tlr $7, T \operatorname{lr} 8$, and Tlr10 (Grabig et al., 2006). Tlr4 is one of the most important pattern recognition receptors that can recognize lipopolysaccharides and heat shock proteins. In turn, Tlr4 engages $M y d 88$, which signals through IRAK4 to turn on a variety of pathways (including $N F-\kappa B$ ) and induces proinflammatory cytokines. Others have reported that probiotics activate $N F-\kappa B$ and stimulate the production of Tnf- $\alpha$ in vitro (Pagnini et al., 2010). $N F-\kappa B$ could induce proinflammatory cytokines such as $I l 6, I l 8$, and $I l 1 \beta$, which play a role in the inflammatory response that is responsible for eliminating pathogens. As in previous studies, in this study, a diet with LGG activated Tlr4-mediated activation of $N F-\kappa B$ and promoted proinflammatory cytokines.

The survival rate of chickens fed with LGG was higher than that of the controls after E. coli infection. This result was supported by increased growth performance, immunoglobulin, and enhanced expression of major innate immunity genes, which are involved in the initiation and regulation of immune response against E. coli. Most importantly, LGG established antimicrobial properties against various pathogenic bacteria by producing bacteriocin, organic acid, and hydrogen peroxide. In the present study, LGG showed strong inhibition against S. aureus, E. coli, S. Paratyphi B, S. Enteritidis, and E. coli in vitro. Meanwhile, LGG prevented colonization and persistence of $E$. coli in chicken intestinal epithelial cells. It is also important 
that LGG can inhibit the pathogenic bacteria by regulating the balance of intestinal microbiota. In addition, the E. coli content in the hearts, kidneys, and lungs significantly declined with a diet of LGG after E. coli infection. As a result, a diet including LGG can inhibit the proliferation of E. coli, enhance the resistance to E. coli invasion of the intestinal tract, and improve the disease process in vivo. Our results indicated that LGG may be a competitive exclusion approach in terms of controlling bacterial infection.

\section{CONCLUSION}

In conclusion, LGG showed great probiotic potential, both in vitro and in vivo. LGG can inhibit the pathogens from adhering to primary chicken intestinal epithelial cells. Chickens fed with LGG exhibited greater growth performance, immunoglobulin concentrations, intestinal health, immune responses, and disease resistance.

\section{DATA AVAILABILITY STATEMENT}

The datasets presented in this study can be found in online repositories. The names of the repository/repositories and accession number(s) can be found below: https://www.ncbi.nlm. nih.gov/sra/?term=PRJNA699761.

\section{REFERENCES}

Abhisingha, M., Dumnil, J., and Pitaksutheepong, C. (2018). Selection of potential probiotic Lactobacillus with inhibitory activity against Salmonella and Fecal Coliform Bacteria. Probiotics Antimicrob. Proteins 10, 218-227. doi: 10.1007/ s12602-017-9304-8

Abrams, G. D., Bauer, H., and Sprinz, H. (1963). Influence of the normal flora on mucosal morphology and cellular renewal in the ileum. A comparison of germ-free and conventional mice. Lab. Invest. 12, 355-364.

Aschfalk, A., and Müller, W. (2002). Clostridium perfringens toxin types from wild-caught Atlantic cod (Gadus morhua L.), determined by PCR and ELISA. Can. J. Microbiol. 48, 365-368. doi: 10.1139/w02-015

Bocourt, R., Savón, L., Díaz, J., Brizuela, M. A., and Elías, A. (2004). Effect of the probiotic activity of Lactobacillus rhamnosus on physiological indicators of suckling pigs. Cuban J. Agric. Sci. 38, 403-408.

Bogovic Matijasić, B., Narat, M., Zoric Peternel, M., and Rogelj, I. (2006). Ability of Lactobacillus gasseri K 7 to inhibit Escherichia coli adhesion in vitro on Caco2 cells and ex vivo on pigs' jejunal tissue. Int. J. Food Microbiol. 107, 92-96. doi: 10.1016/j.ijfoodmicro.2005.08.017

Fernández, M. F., Boris, S., and Barbés, C. (2003). Probiotic properties of human lactobacilli strains to be used in the gastrointestinal tract. J. Appl. Microbiol. 94, 449-455. doi: 10.1046/j.1365-2672.2003.01850.x

Fernández de Palencia, P., López, P., Corbí, A. L., Peláez, C., and Requena, T. (2008). Probiotic strains: survival under simulated gastrointestinal conditions, in vitro adhesion to Caco-2 cells and effect on cytokine secretion. Eur. Food Res. Technol. 227, 1475-1484. doi: 10.1007/s00217-008-0870-6

Forder, R. E., Howarth, G. S., Tivey, D. R., and Hughes, R. J. (2007). Bacterial modulation of small intestinal goblet cells and mucin composition during early posthatch development of poultry. Poult. Sci. 86, 2396-2403. doi: 10.3382/ps. 2007-00222

Gao, J., Li, Y., Wan, Y., Hu, T., Liu, L., Yang, S., et al. (2019). A novel postbiotic from Lactobacillus rhamnosus GG With a beneficial effect on intestinal barrier function. Front. Microbiol. 10:477. doi: 10.3389/fmicb.2019.00477

Grabig, A., Paclik, D., Guzy, C., Dankof, A., Baumgart, D. C., Erckenbrecht, J., et al. (2006). Escherichia coli strain Nissle 1917 ameliorates experimental colitis

\section{ETHICS STATEMENT}

The animal study was reviewed and approved by the Committee on the Ethics of Animal Experiments of Yangzhou University.

\section{AUTHOR CONTRIBUTIONS}

MG designed and investigated the study, and wrote the original draft. CoZ carried out the verification and data analysis. ChZ and $\mathrm{XZ}$ discussed the results. YW reviewed and edited the manuscript. All authors contributed to the article and approved the submitted version.

\section{FUNDING}

This work was supported by the National Natural Science Foundation of China (31902236), the Natural Science Foundation of the Jiangsu Higher Education Institutions of China (19KJB230002), the National Key Research and Development Project of China (2016YFD0501609), the China Postdoctoral Science Foundation (2019M651987), and the Priority Academic Program Development of Jiangsu Higher Education Institutions.

via toll-like receptor 2- and toll-like receptor 4-dependent pathways. Infect. Immun. 74, 4075-4082. doi: 10.1128/iai.01449-05

Guo, S., Li, C., Liu, D., and Guo, Y. (2015). Inflammatory responses to a Clostridium perfringens type A strain and $\alpha$-toxin in primary intestinal epithelial cells of chicken embryos. Avian Pathol. 44, 81-91. doi: 10.1080/ 03079457.2015.1005573

He, X., Zeng, Q., Puthiyakunnon, S., Zeng, Z., Yang, W., Qiu, J., et al. (2017). Lactobacillus rhamnosus GG supernatant enhance neonatal resistance to systemic Escherichia coli K1 infection by accelerating development of intestinal defense. Sci. Rep. 7:43305.

Hwanhlem, N., Ivanova, T., Biscola, V., Choiset, Y., and Haertlé, T. (2017). Bacteriocin producing Enterococcus faecalis isolated from chicken gastrointestinal tract originating from Phitsanulok, Thailand: isolation, screening, safety evaluation and probiotic properties. Food Control 78, 187-195. doi: 10.1016/j.foodcont.2017.02.060

Kirjavainen, P. V., Ouwehand, A. C., Isolauri, E., and Salminen, S. J. (1998). The ability of probiotic bacteria to bind to human intestinal mucus. FEMS Microbiol. Lett. 167, 185-189. doi: 10.1111/j.1574-6968.1998.tb13 226.x

Lönnermark, E., Nowrouzinan, F., Adlerberth, I., Ahrné, S., Wold, A., and Friman, V. (2012). Oral and faecal lactobacilli and their expression of mannose-specific adhesins in individuals with and without IgA deficiency. Int. J. Med. Microbiol. 302, 53-60. doi: 10.1016/j.ijmm.2011.08.004

Lupindu, A. M., Dalsgaard, A., Msoffe, P. L., Ngowi, H. A., Mtambo, M. M., and Olsen, J. E. (2015). Transmission of antibiotic-resistant Escherichia coli between cattle, humans and the environment in peri-urban livestock keeping communities in Morogoro, Tanzania. Prev. Vet. Med. 118, 477-482. doi: 10. 1016/j.prevetmed.2014.12.005

Ng, S. C., Hart, A. L., Kamm, M. A., Stagg, A. J., and Knight, S. C. (2009). Mechanisms of action of probiotics: recent advances. Inflamm. Bowel Dis. 15, 300-310. doi: 10.1002/ibd.20602

Pagnini, C., Saeed, R., Bamias, G., Arseneau, K. O., Pizarro, T. T., and Cominelli, F. (2010). Probiotics promote gut health through stimulation of epithelial innate immunity. Proc. Natl. Acad. Sci. U.S.A. 107, 454-459. doi: 10.1073/pnas. 0910307107 
Pereira, R., Bortoluzzi, C., Durrer, A., Fagundes, N. S., Pedroso, A. A., Rafael, J. M., et al. (2019). Performance and intestinal microbiota of chickens receiving probiotic in the feed and submitted to antibiotic therapy. J. Anim. Physiol. Anim. Nutr. 103, 72-86. doi: 10.1111/jpn. 13004

Pradhan, D., Mallappa, R. H., and Grover, S. (2020). Comprehensive approaches for assessing the safety of probiotic bacteria. Food Control 108:106872. doi: 10.1016/j.foodcont.2019.106872

Rashid, M. U., Weintraub, A., and Nord, C. E. (2012). Effect of new antimicrobial agents on the ecological balance of human microflora. Anaerobe 18, 249-253. doi: 10.1016/j.anaerobe.2011.11.005

Savijoki, K., Lietzén, N., Kankainen, M., Alatossava, T., Koskenniemi, K., Varmanen, P., et al. (2011). Comparative proteome cataloging of Lactobacillus rhamnosus strains GG and Lc705. J. Proteome Res. 10, 3460-3473. doi: 10.1021/ pr2000896

Simoneit, C., Burow, E., Tenhagen, B. A., and Käsbohrer, A. (2014). Oral administration of antimicrobials increase antimicrobial resistance in E. coli from chicken - A systematic review. Prev. Vet. Med. 118, 1-7. doi: 10.1016/ j.prevetmed.2014.11.010

Stanley, D., Denman, S. E., Hughes, R. J., Geier, M. S., Crowley, T. M., Chen, H., et al. (2012). Intestinal microbiota associated with differential feed conversion efficiency in chickens. Appl. Microbiol. Biotechnol. 96, 1361-1369. doi: 10.1007/ s00253-011-3847-5

Wang, Y., Gong, L., Wu, Y. P., Cui, Z. W., Wang, Y. Q., Huang, Y., et al. (2019). Oral administration of Lactobacillus rhamnosus GG to newborn piglets augments gut barrier function in pre-weaning piglets. J. Zhejiang Univ. Sci. B 20, 180-192. doi: 10.1631/jzus.b1800022

Yan, F., Liu, L., Dempsey, P. J., Tsai, Y. H., Raines, E. W., Wilson, C. L., et al. (2013). A Lactobacillus rhamnosus GG-derived soluble protein, p40, stimulates ligand release from intestinal epithelial cells to transactivate epidermal growth factor receptor. J. Biol. Chem. 288, 30742-30751. doi: 10.1074/jbc.m113. 492397

Zhang, D., Li, R., and Li, J. (2012). Lactobacillus reuteri ATCC 55730 and L22 display probiotic potential in vitro and protect against Salmonella-induced pullorum disease in a chick model of infection. Res. Vet. Sci. 93, 366-373. doi: 10.1016/j.rvsc.2011.06.020

Zhang, J. L., Xie, Q. M., Ji, J., Yang, W. H., Wu, Y. B., Li, C., et al. (2012). Different combinations of probiotics improve the production performance, egg quality, and immune response of layer hens. Poult. Sci. 91, 2755-2760. doi: $10.3382 /$ ps.2012-02339

Zhang, J. M., Sun, Y. S., Zhao, L. Q., Chen, T. T., Fan, M. N., Jiao, H. C., et al. (2019). SCFAs-induced GLP-1 secretion links the regulation of gut microbiome on hepatic Lipogenesis in Chickens. Front. Microbiol. 10:2176. doi: 10.3389/ fmicb.2019.02176

Zhang, L., Xu, Y. Q., Liu, H. Y., Lai, T., Ma, J. L., Wang, J. F., et al. (2010). Evaluation of Lactobacillus rhamnosus GG using an Escherichia coli K88 model of piglet diarrhoea: effects on diarrhoea incidence, faecal microflora and immune responses. Vet. Microbiol. 141, 142-148. doi: 10.1016/j.vetmic.2009. 09.003

Conflict of Interest: The authors declare that the research was conducted in the absence of any commercial or financial relationships that could be construed as a potential conflict of interest.

Copyright (c) 2021 Guo, Zhang, Zhang, Zhang and Wu. This is an open-access article distributed under the terms of the Creative Commons Attribution License (CC BY). The use, distribution or reproduction in other forums is permitted, provided the original author(s) and the copyright owner(s) are credited and that the original publication in this journal is cited, in accordance with accepted academic practice. No use, distribution or reproduction is permitted which does not comply with these terms. 